caused by diverticulitis, carcinoma colon. Ureterocolonic fistulas are caused by the inflammatory condition of the urinary system or colon like an untreated perinephric abscess, calculus pyonephrosis.

Conclusions It was a very rare case of Ureteroileal fistula which developed post laparoscopic surgery trauma in a young girl, Patient responded well to conservative treatment.

\section{IDDF2019-ABS-0206 CARCINOMA PENIS WITH UNUSUAL SITE OF METASTASIS TO LIVER AND AT TIP OF NOSE}

Vijay Sharma*, Richa Sharma, Dinesh Gupta. Regional institute of health medicine and research, Jaipur, Rajasthan, India

\subsection{6/gutjnl-2019-IDDFabstracts.197}

Background Carcinoma of the penis is an uncommon malignancy in Western countries, representing $0.4 \%$ of male malignancies, while in Asia, Africa, and South America 10\% of all malignancies. The most common distant metastatic sites are the lung, bone, and liver. Local recurrence after a properly planned and executed partial or total penectomy is rare. However local recurrences may occur at the remaining shaft of the penis or at neomeatus or in subcutaneous nodules in nearby skin of groin after groin node dissection. However, recurrences in subcutaneous nodules at distant sites have not been seen in literature. We present here a case of carcinoma penis, treated with total penectomy and bilateral ilio-inguinal block dissection presenting with jaundice, the suggestion of liver metastasis and subcutaneous recurrence.

Methods 60 years old, non-smoker male with no co-morbid conditions presented with a history of a small ulcer over penis for 3-4 months, underwent circumcision. He underwent total penectomy with bilateral ilio-inguinal block dissection. Biopsy \& Histopathology was suggestive of moderately differentiated squamous cellcarcinom. Few inguinal and pelvic nodes were positive for metastasis. He was advised adjuvant treatment (radiotherapy) but defaulted for $\underline{3}$ months. Then he presented with multiple small nodules in left grow in region suggestive of recurrence. Palliative radiotherapy was started to inguinal region. During radiotherapy, a subcutaneous nodule developed over the tip of the nose. (figure 1) Biopsy from the nodule was positive for metastatic squamous cell carcinoma.

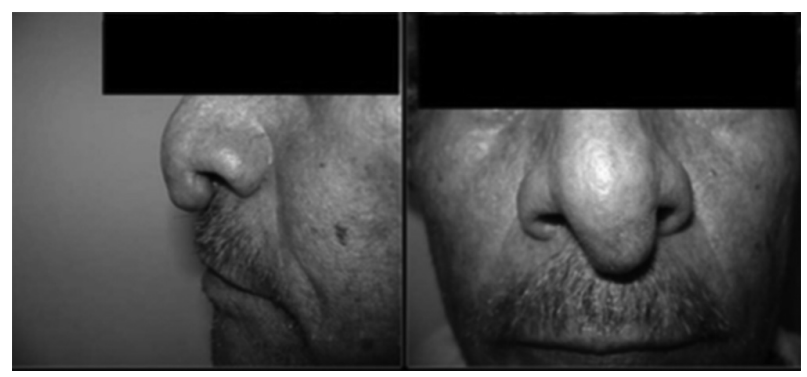

Abstract IDDF2019-ABS-0206 Figure 1 Metastasis at tip of nose
Results Cases of cutaneous metastasis to penis have also been mentioned in literature from various organs like urothelial carcinomas, colorectal adenocarcinomas, pulmonary carcinomas, squamous cell carcinoma of the base tongue, cutaneous malignant melanoma and acute myeloid leukemia. A case of distant cutaneous metastasis from penis has not been mentioned in literature to the best of our knowledge.

Conclusions This patient developed local recurrence in the form of subcutaneous groin nodule, liver metastasis and a distant metastatic subcutaneous nodule at the tip of the nose. This case is presented because of the unusual and rare presentation in the form of distant subcutaneous metastasis.

\section{IDDF2019-ABS-0214 PAIN REDUCTION IN LICHTENSTEIN OPEN INGUINAL HERNIA REPAIR USING LIGHT WEIGHT PROLENE MESH GRAFT ON UNILATERAL GROIN HERNIA}

${ }^{1}$ Budhi Ida Bagus*, ${ }^{2}$ Ahmad Fandi. 'Department of Surgery, Sebelas Maret University, Indonesia; ${ }^{2}$ Department of Surgery, Moewardi General Hospital, Indonesia

\subsection{6/gutjnl-2019-IDDFabstracts. 198}

Background More than 20 million patients undergo groin hernia repair annually. The many different approaches, treatment indications and a significant array of techniques for groin hernia repair warrant guidelines to standardize care, minimize complications, and improve results. Surgical treatment is successful in the majority of cases, but recurrences necessitate reoperations in $10-15 \%$ and long-term disability due to chronic pain (pain lasting longer than 3 months) occurs in $10-12 \%$ of patients. Approximately $1-3 \%$ of patients have severe chronic pain. There were not enough guidelines recommended on choosing the type of prolene mesh for Lichtenstein open inguinal hernia repair.

Methods This was a prospective study, which evaluated 25 cases of a unilateral inguinal hernia on both sex whose performed Lichtenstein open inguinal hernia repair using heavy and light weight prolene mesh from January till December 2018, divided into 2 groups. Post operative pain, chronic pain, time of return to daily activity and the evidence of recurrence were evaluated and recorded. 3 months follow up was done, the subject whose can not evaluate during this period of time was being excluded.

Results During 3 months follow up between 2 groups (13 cases using heavy weight mesh and 12 cases using light weigh prolen mesh), there was no recurrence found on those groups. Post operative pain was the same between 2 groups. Chronic pain was less on light weight mesh hernia repair with VAS (visual analog score) 1-2 after 3 months compared with heavy weight mesh group $(\mathrm{p}<.005)$. Return to daily activity was shorter on light weight mesh group (2 vs 5 days, $\mathrm{p}<.005)$.

Conclusions Light weight prolene mesh associated with a reduction in post operative pain after Lichtenstein Open Inguinal Hernia Repair and earlier time of return to daily activity.

Pain, light weight prolene mesh, unilateral groin hernia 